\title{
Dopant ions size impact on structural properties of ordered poly(3,4-ethylenedioxythiophene) systems $\left.{ }^{*}\right)$
}

\author{
Maciej Śniechowski ${ }^{1) * *)}$, Tomasz A. Kozik ${ }^{1)}$, Wojciech Łużny ${ }^{1)}$ \\ DOI: dx.doi.org/10.14314/polimery.2020.9.6
}

\begin{abstract}
The purpose of this work was to determine whether the structures of ordered part of novel poly(3,4-ethylenedioxythiophene) PEDOT:OTf (doped with trifluoromethanesulfonate anions) and PEDOT:HSulf (doped with hydrogensulfate anions) systems could really be simply the layered structure of PEDOT: Tos (doped with tosylate anions) with the counter ion changed to the appropriate one. Insight into this problem could be provided by molecular dynamics simulations but also by swarm intelligence optimization, developed earlier for the purpose of investigating the other structures.
\end{abstract}

Keywords: self-organization, molecular dynamics simulations, conductive polymers, X-ray diffraction, crystal structure

\section{Wpływ wielkości jonów domieszkujących na właściwości strukturalne uporządkowanych układów poli(3,4-etylenodioksytiofenu)}

Streszczenie: Zbadano zdolność struktury uporządkowanej części nowych układów domieszkowanych poli(3,4-etylenodioksytiofenu) (PEDOT) [PEDOT : OTf (domieszkowane anionami trifluorometanosulfonianowymi) i PEDOT: HSulf (domieszkowane anionami wodorosiarczanowymi)] do powielania znanej z literatury warstwowej struktury PEDOT: Tos (domieszkowane anionami tosylanowymi) z jonem domieszki zmienionym na odpowiedni. Poszukiwania nowych struktur wspomagano symulacjami dynamiki molekularnej, a także z zastosowaniem optymalizacji opartej na algorytmach inteligencji stadnej.

Słowa kluczowe: samoorganizacja, symulacje dynamiki molekularnej, polimery przewodzące, dyfrakcja promieniowania rentgenowskiego, struktura krystaliczna.

Recently, some new doped PEDOT [poly(3,4-ethylenedioxythiophene)] systems exhibiting very high conductivity and excellent thermoelectric properties have been developed [1-3]. One of the developed systems was synthesized by adding iron(III) trifluoromethanesulfonate to the polymerization solution. The resulting system contained electron deficient PEDOT chains and trifluoromethanesulfonate counter ions, also commonly called triflate anions and abbreviated OTf- or simply OTf. The obtained novel conducting polymer system was named in an abbreviated manner PEDOT: OTf by Massonnet et al. [1]. The result of the treatment the synthesized polymer system with sulfuric acid was a significant increase in the conductivity of the sample. Very interestingly, it was also found by Xia et al. [4] that the treatment of PEDOT: PSS [poly(3,4-ethylenedioxythiophene)-

\footnotetext{
1) Faculty of Physics and Applied Computer Science, AGH University of Science and Technology, Al. Mickiewicza 30, 30-059 Cracow, Poland.

*) Material contained in this article was presented at the XI International Conference on "X-ray Investigations of Polymer Structure", 3-6 December 2019, Ustroń, Poland.

**) Author for correspondence: sniechowski@fis.agh.edu.pl
}

poly(styrenesulfonate)] samples with sulfuric acid leads to a large increase in the conductivity of the sample.

It was concluded by Xia et al. that some of the PSS polyanions are replaced by hydrogen sulfate ions. However, Massonnet et al. stated, basing on the results of different experimental measurements, that the PEDOT: OTf system underwent a large scale or even complete ion exchange and most of the triflate ions were replaced by the hydrogen sulfate ions, even naming the obtained polymer system PEDOT: HSulf.

The measurements the X-ray diffraction for both systems PEDOT: OTf and PEDOT: HSulf yielded similar

XRD patterns clearly containing four distinguished Bragg peaks. The studied systems were partially crystalline as indicated by TEM images shown in [2].

\section{MOLECULAR DYNAMICS MODELS OF THE CONSTITUENTS OF THE STUDIED SYSTEMS}

The GROMACS package used in MD (molecular dynamics) simulations does not include topological models for PEDOT mers nor for the dopants studied in this work. This is why topological entries had to be added to the internal database of the package. Besides calculating 
partial charges, some adjustments to the potentials had to be made.

\section{Partial charge models for PEDOT macromolecules} and for tosylate, triflate, hydrogen sulfate and sulfate ions

Formulating a partial charge model for a polymer requires taking into account the fact that the first and last mer unit (repeating unit) within the chain may have a different electrostatic potential distribution in their vicinity compared to a mer unit from the middle of the chain. To take this into account, DFT (Density-Functional Theory) calculations should be performed for oligomers of the studied polymer (definitely not for the monomer). It was decided to use tetramers of PEDOT for the sake of designating partial charges, since the two mer units which were not the terminal ones exhibited a partial charge distribution [according to ESP (Electro-Static Potential) fitting] close to a symmetrical one. Introducing constraints for partial charges in the ESP fitting procedure to enforce full symmetry was thus not considered a large alteration of the electrostatics of the actual molecule.

DFT calculations using the B3LYP exchange-correlation functional and the $6-311 G^{* *}$ Gaussian basis were performed in NWChem for tetramers of PEDOT with a zero net charge (pristine), with a $+1 e$ net charge $(+0.25 e$ per unit, constrained) and with a $+2 e$ net charge $(+0.5 e$ per unit, constrained). This was done to take into account different doping ratios in further studies. A graphical depiction of the tetramer along and dopant ions with atom labels are shown in Fig. 1 and Fig. 2.

The partial charges obtained by ESP fitting to the DFT calculation results for the repeating unit (mer unit) (terminal groups are absent in MD simulations due to periodic condition) and for counter ions were shown in [5]. It was assumed that an acceptable approximation of the partial charge model for longer chains was a model in

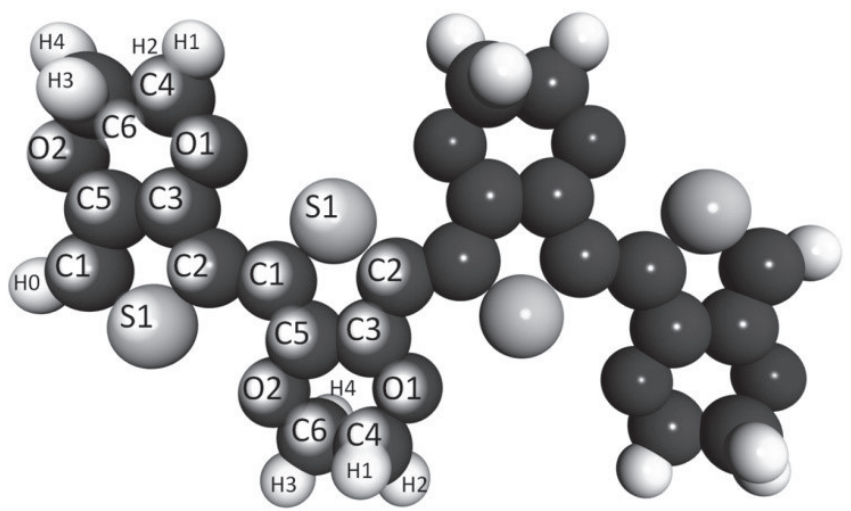

Fig. 1. Graphical (3D rendered) depiction of the studied tetramer of PEDOT along with atom labels; the molecule and labels (for unlabeled atoms) exhibit rotation symmetry by the axis perpendicular to the image and to the bond between atom $\mathrm{C} 2$ and the first unlabeled carbon atom which all the repeating units have partial charges of the repeating unit of the tetramer, regardless of the length of the entire polymer. This approach was used in [6].

\section{Choice of potentials for the PEDOT model and for the ion models}

The OPLS-AA force field, which was chosen for simulations, included most of the potentials needed to model a PEDOT chain. The reference [6] includes a study of polythiophene, where some issues regarding missing potentials were resolved. Because PEDOT differs from thiophene only by the additional groups bound to the aromatic system, some solutions from the cited reference were reused for the purpose of this study. The most important ones were the dihedral potentials for the torsion angle between polythiophene rings, derived by K. Piwowarczyk for bithiophene. These potentials override the default values coincidently included in the OPLS-AA force field, intended for non-polymeric molecules. GROMACS uses a non-redundant set of atom types for mapping OPLS atom types internally. This typization is not absolute, as indicated by frequent comments within the database. A lot of entries (especially for bond angle potentials) are followed by a comment that the potential is a copy of a potential in which the type of, i.e. one atom is of a formally different type or that the potential has been adjusted from a similar potential. The OPLS-AA force field in the GROMACS internal database was adjusted in this manner to handle PEDOT molecules. All the modifications and extensions made for this purpose, for harmonic bond potentials, for harmonic bond angle potentials and for dihedral potentials were shown in [5].

\section{Prepared crystalline models}

Crystalline models were generated using own GenGROTOP [5] program, which was upgraded to support PEDOT chains topologically linked through one of the periodic boundaries. The program was also modified to support tosylate, triflate, hydrogen sulfate and sulfate ions. One model of a PEDOT: Tos crystallite was prepared. It consisted of 6 layers of stacked PEDOT chains,

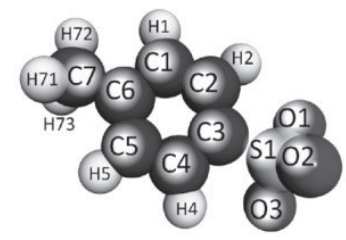

b)

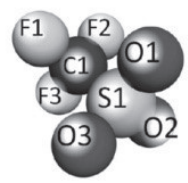

c)

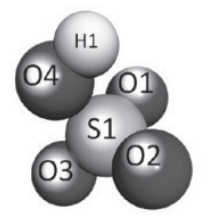

Fig. 2. Graphical (3D rendered) depictions of the studied ions along with atom labels: a) tosylate ion, b) triflate ion, c) hydrogen sulfate ion; sulfate ion only lacks the hydrogen atom compared to c) 
alternating with 6 layers of tosylate anions. Each layer of PEDOT consisted of 22 chains, each containing 18 mers. The layout was in accordance with the structure described in [7]. The doping ratio of $25 \%$ assumed in the cited reference required organizing the counter ions in 6 rectangular layers, each in the form of a $11 \times 9$ matrix.

The model underwent an energy minimization procedure, after which a relaxation simulation was performed. It consisted of 2000000 steps with a time step of 1 fs. Initial velocities were generated from a Maxwell distribution at $300 \mathrm{~K}$. Electrostatic and van der Waals interactions were handled using the PME (Particle-Mesh Ewald) method. A cut off distance for short range non bonded interactions of $3 \mathrm{~nm}$ was used. The neighbor list was updated every 50 steps. The temperature was coupled to $300 \mathrm{~K}$ using a velocity rescale thermostat with a coupling constant equal to 1 ps every time step. The pressure was coupled to $0.1 \mathrm{MPa}$ (1 bar) using an anisotropic Berendsen type barostat applied every step, with a coupling constant of 0.2 ps and a compressibility of $4.5 \cdot 10^{-10} 1 / \mathrm{Pa}$ $\left(4.5 \cdot 10^{-5} 1 /\right.$ bar $)$ for each direction.

The model of PEDOT: OTf crystallites was prepared as follows. The large model, used for structural investigation, consisted of 8 layers of PEDOT chains alternating with 8 layers of triflate anions. Each PEDOT layer contained 30 stacked chains, each consisting of 26 mers. The starting structure was constructed in accordance with the model used for PEDOT: Tos, with the counter ions changed to triflate ones. The model underwent an energy minimization procedure, followed by a relaxation simulation consisting of 4000000 steps with a time step of $0.5 \mathrm{fs}$. After a correction of the used potential which contained a minor error, another 1000000 steps with a time step of $1 \mathrm{fs}$ were performed. The settings were the same as in the case of the PEDOT: Tos model, the differences being the cut off distances which were all set to $4.5 \mathrm{~nm}$.

The model of PEDOT: HSulf crystallites was prepared in nearly identical procedure as in the case of PEDOT: OTf, the difference being that the potential was correct from the start so the additional 1 ns equilibration simulation was not performed for the model. However, for investigation purposes both models were simulated again with identical settings, but with different starting structures. Instead of using structures generated by GenGROTOP, the relaxed structures of the PEDOT: OTf models of matching size with counter ions changed from triflate to hydrogen sulfate ones were used as starting frames for the simulations. The reason for doing so will be described further.

One hypothetical model of a doped PEDOT system with sulfate anions as counter ions was prepared, further referred to as PEDOT:Sulf. It was prepared in the same manner as the large PEDOT: OTf model. To avoid a different ion number and layout, a plausible doping ratio of $50 \%$ was assumed. The starting unit cell generated by GenGROTOP had thanks to this an identical layout as earlier models, but used a different charge model of $+0.5 e$ charge per mer instead of $+0.25 e$ charge per mer. Similarly to the earlier models of PEDOT: HSulf, the large model of PEDOT: Sulf was simulated again using the structure of the relaxed, large PEDOT: OTf crystallite with triflate ions changed to sulfate ions as the starting structure and charge model adjusted to reflect the different doping ratio. This was done for structural investigation purposes, described further in the text.

\section{RESULTS AND DISCUSSION}

\section{Crystalline structure of PEDOT: Tos as a verification of the force field}

In the layers of stacked PEDOT chains, the chains were alternatingly, slightly shifted upwards or downwards to accommodate the $\mathrm{SO}_{3}$ - groups of Tos ions. This subtle effect, computed by Kim et al. [8] at DFT level of theory, was reproduced in simulations thanks to the point partial charge model being based on an ESP fit to the DFT charge density. The PEDOT chains were nearly planar, with very minor out of plane rotations of the aromatic groups. The two $\mathrm{CH}_{2}$ groups in each mer appeared to exhibit some more flexibility, slightly bending out of the chain planes. The inter layer distance in the simulated model was ca. $13.9 \AA$, which is in agreement with the value of $14 \AA$ in both of the literature unit cell models.

The very good agreement of the simulation with literature unit cell models of PEDOT : Tos (tosylate) system verified that the prepared MD model of PEDOT, which was a composition of several adjusted OPLS-AA potentials, was correct and could be used for further simulations. The final frame of the simulation of the only PEDOT : Tos model viewed along the polymer chain axes, is shown below in Fig. 3.

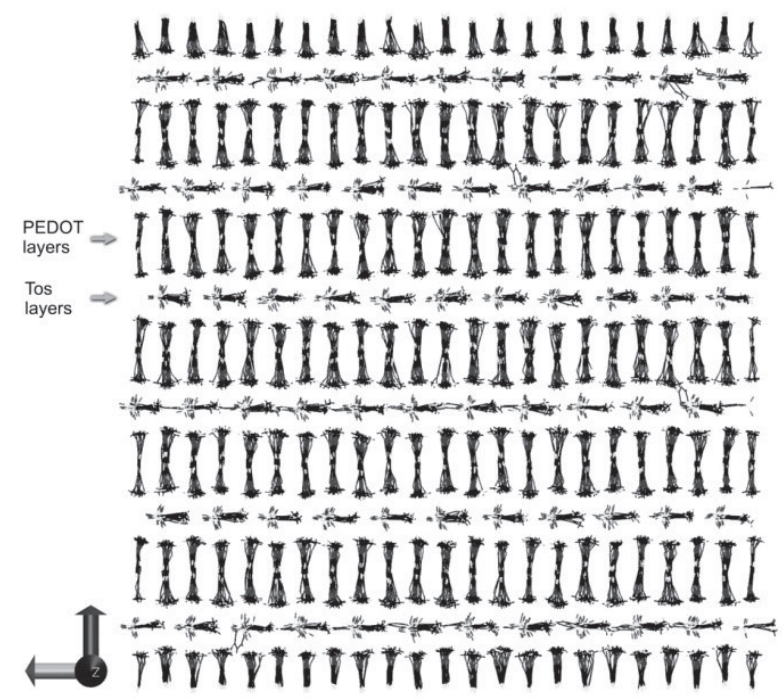

Fig. 3. The final frame of the PEDOT: Tos model (view of simulation periodic box along $P E D O T$ chain axes) the arrows indicate Cartesian coordinates 


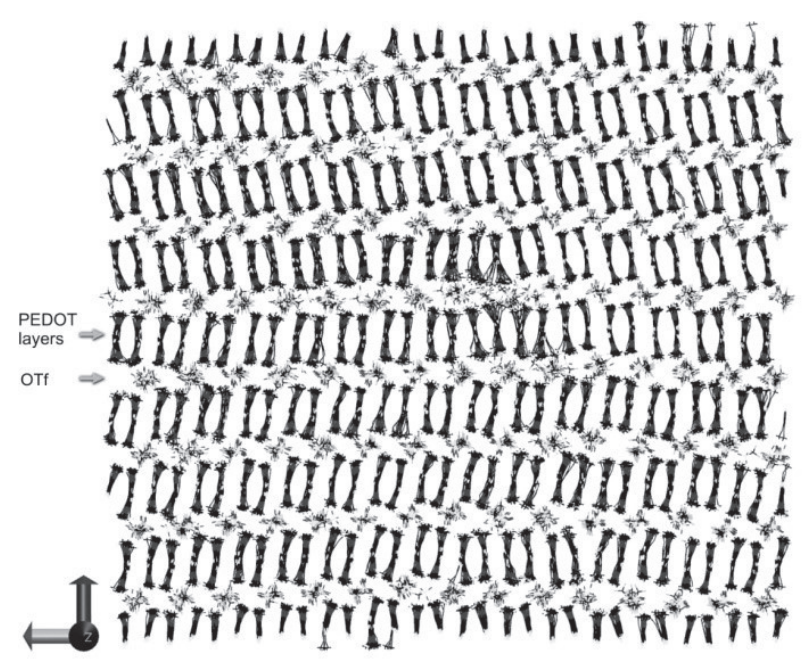

Fig. 4. The final frame of the PEDOT: OTf large model (view of simulation periodic box along PEDOT chain axes) the arrows indicate Cartesian coordinates

\section{Crystalline structure of PEDOT: OTf}

Simulations results show that PEDOT: Tos model of Aasmudtveit et al. [7] with tosylate counter ions replaced with triflate counter ions is not a stable system. The final frame of the simulation of the large PEDOT: OTf crystallite model, viewed along the polymer chain axes, is shown in Fig. 4.

The PEDOT layers were not only closer to each other, but also distorted by the counter ions. Namely, the

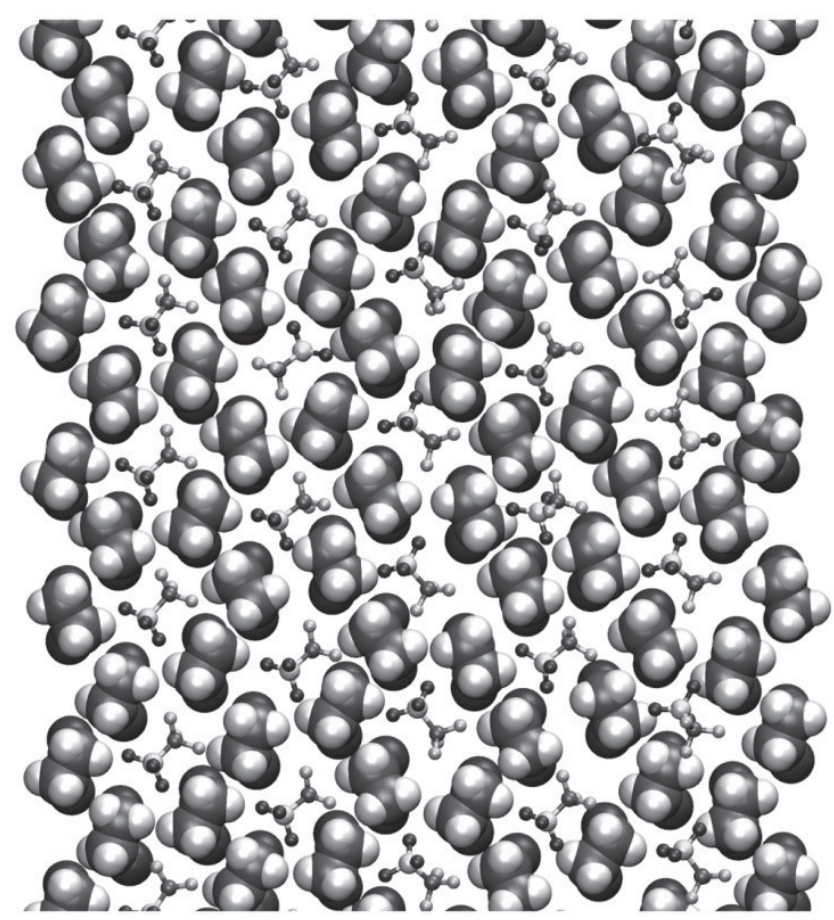

Fig. 5. The structure of a triflate ion layer in the PEDOT:OTf system; the ball-and-stick models represent triflate ions; the large van der Waals spheres represent the $-\mathrm{O}-\mathrm{CH}_{2}-\mathrm{CH}_{2}-\mathrm{O}-$ fragments of PEDOT chains from the layer below tighter packing of the structure systematically caused a bending of the bound $\mathrm{CH}_{2}$ groups of the polymer chain out of its plane. This had a side effect of pairing neighboring chains, which seemed to exhibit the sandwichtype stacking relatively to each other, but a stacking type closer to the parallel-displaced type relatively to other pairs. The triflate ions within the layer are organized into a pseudo-hexagonal (pseudo due to the coincidental close match between its characteristic distances along the chains and across the chains) structure rather than a rectangular one. What is more, the $\mathrm{CH}_{2}$ groups of the PEDOT chains seemed to have adjusted to this structure and formed small, distorted hexagonal "pockets" in which the counter ions could reside. This structure also enabled stronger interaction between $\mathrm{CH}_{2}$ groups from the PEDOT layer below the ion layer and above it. The $\mathrm{CH}_{2}$ groups from consecutive layers almost touched, while in the PEDOT: Tos structure they were well separated by the counter ion layers.

To better understand the interactions between the polymer layers and the triflate anions, the structure of the counter ion layers was investigated. Initially, in the starting structure, the ions in the layers formed a rectangular $11 \times 9$ structure. In the final frames, the structure was very different. The effect of energy minimization and packing optimization was a shallow penetration of the polymer layers by the triflate ions. While it seemed that the ions were not small enough to fit between a mer and its next nearest neighboring mer, they managed to enforce a movement of the polymer chains, which could easily slide against each other, as indicated by the simulation result. A visualization of a fragment of the structure of the triflate layers along with how the $\mathrm{CH}_{2}$ groups of a PEDOT chain organize around them is shown in Fig. 5.

To provide additional insight into the crystalline structure of PEDOT:OTf, the X-ray diffraction pattern of the

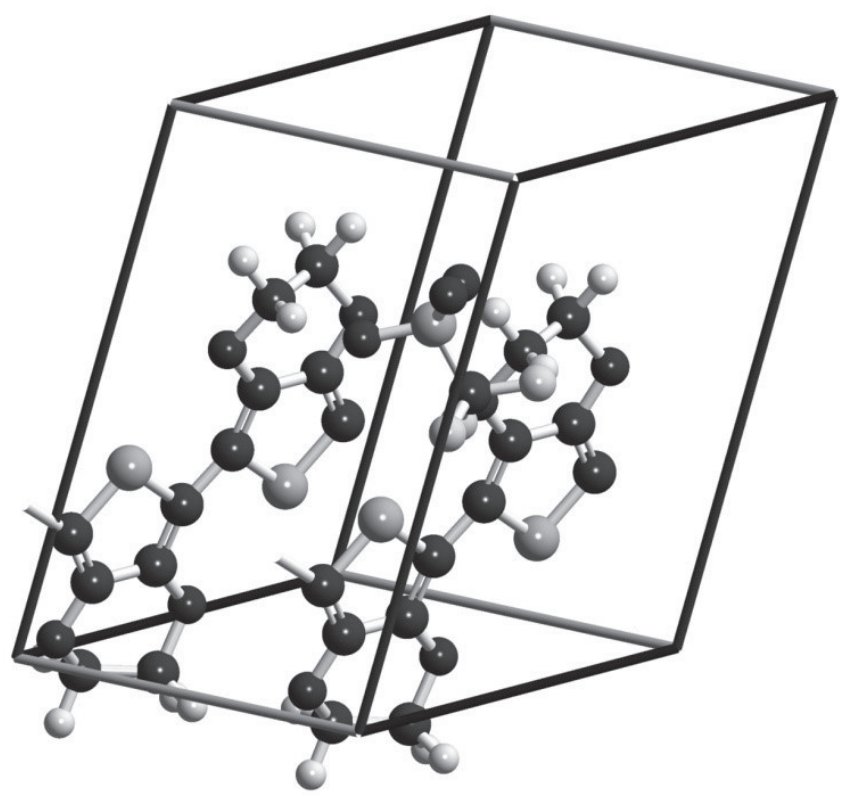

Fig. 6. The best unit cell of PEDOT: OTf found by the optimizer 


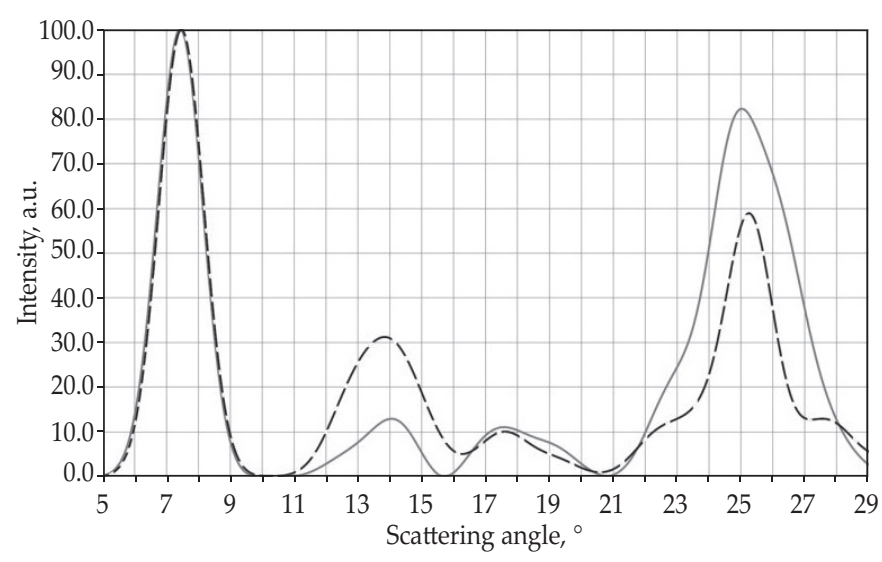

Fig. 7. Calculated powder XRD pattern of the best PEDOT: OTf unit cell found by the optimizer (dashed line) versus the reference pattern (solid line)

model was calculated using the formulas described in [9] applied to the entire simulation box treated as a unit cell. The experimental X-ray diffraction pattern published in [1] in Fig. 5a and the one obtained for the simulation box exhibited a very good qualitative agreement when considering the overall shape, including the small bump on the left slope of the fourth peak. While slight differences in peak positions caused most probably by the idealization of periodic boundary conditions in the simulation did exist, the structure definitely captured fundamental structural aspects of the crystalline phase of the PEDOT: OTf system. The computed from simulation XRD pattern was used as a basis for swarm intelligence based optimization aimed at finding a model of the unit cell of the crystalline phase [10]. The principles behind formulating a detailed modeling scope and typical optimization algorithm settings were presented in

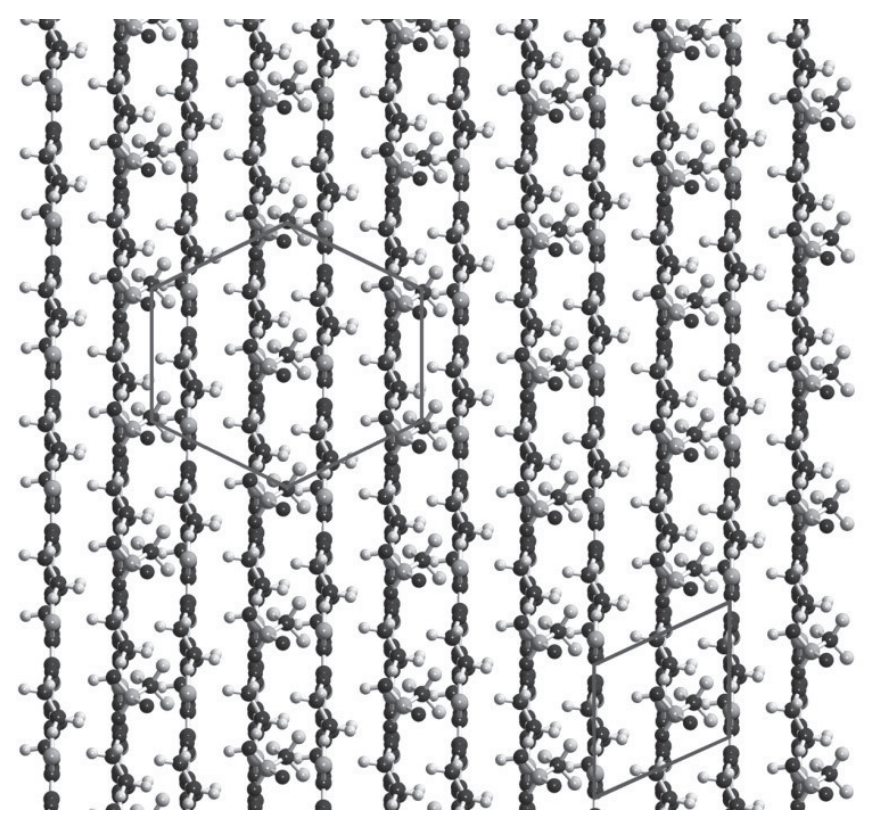

Fig. 8. Optimized PEDOT:OTf structure; layer plane coplanar with the page; outlined unit cell; marked hexagon formed by OTf ions
[5, 9]. A visualization of the optimized PEDOT: OTf unit cell is shown in Fig. 6.

The calculated XRD pattern of the model, compared to the reference XRD pattern extracted from the pattern calculated for the simulation box, is shown in Fig. 7.

The scattering vector magnitude was converted to scattering angle assuming a $\mathrm{Cu} \cdot \mathrm{K \alpha}$ wavelength. The fit of the pattern was very reasonable. It should be pointed out that a lot of disorder elements were omitted, most notably the high distortion of the hexagonal pockets and the varying orientation of triflate ions. The first and third peak were fitted almost perfectly, the second peak was overestimated most probably due to the disorder impact on its intensity in the real structure and the fifth peak was slightly underestimated. The last mismatch could be reduced by improving the formula for calculating the reflex width, which could merge the three peaks forming the reflex. A visualization of the structure obtained by applying translational symmetry to the unit cell model viewed in the direction perpendicular to the layer plane is shown below in Fig. 8.

The reproduction of the pseudo-hexagonal arrangement is clear in such a view. An interesting visualization of the structure viewed along the chain axes is shown below in Fig. 9.

The tilt of the PEDOT chains visible in Fig. 4. was also reproduced, although perhaps in an exaggerated manner.

\section{Crystalline structure of PEDOT: HSulf}

The final frame of the simulation of the large PEDOT: HSulf crystallite model, viewed along the polymer chain axes, is shown below in Fig. 10.

Again, the PEDOT: Tos model of Aasmudtveit et al. [7] with tosylate counter ions replaced with hydrogen sulfate counter ions was not a stable system. The inter layer distance of approximately $12.2 \AA$ was again smaller than in the PEDOT: Tos model (13.9 $\AA$ ), which again indicated a contraction in the direction perpendicular to the layers. The distance was slightly larger than for PEDOT: OTf. Contrarily to PEDOT: OTf however, the PEDOT layers

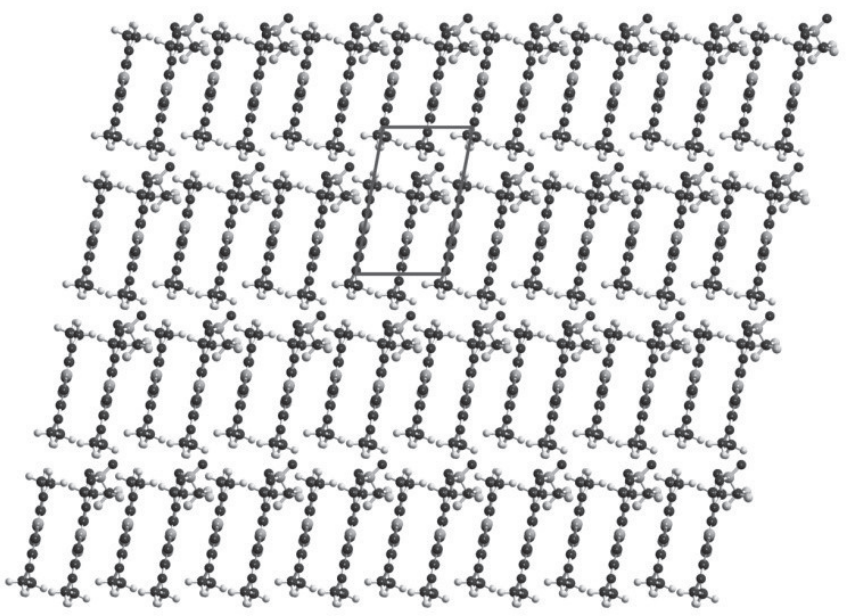

Fig. 9. Optimized PEDOT: OTf structure viewed along the polymer chain axes; outlined unit cell 


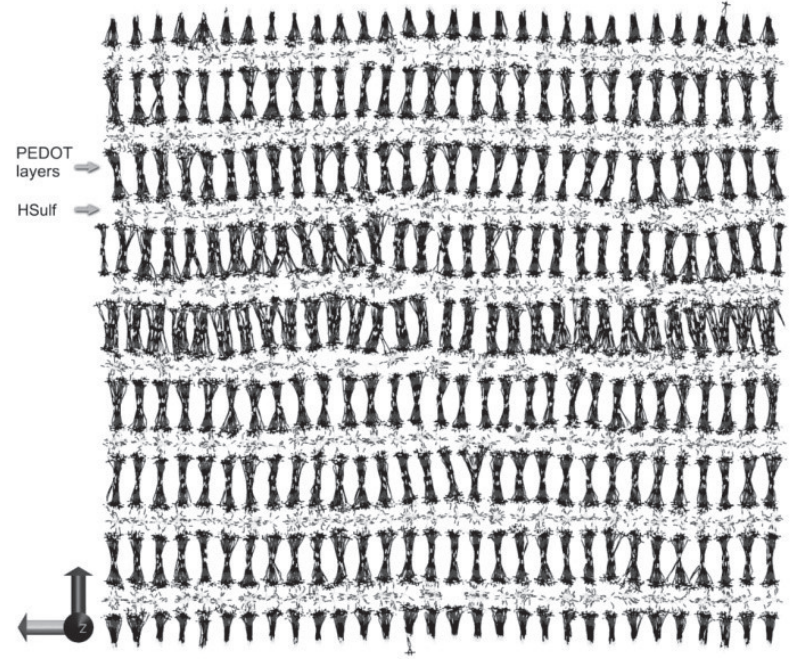

Fig. 10. The final frame of the PEDOT: HSulf large model (view of simulation periodic box along PEDOT chain axes) the arrows indicate Cartesian coordinates

were not distorted by the counter ions as much. The polymer chains also did not form slightly tilted pairs.

In light of molecular dynamics simulations, both a rectangular and a pseudohexagonal structure of PEDOT: HSulf appeared to be stable (Fig. 11).

Simulations thus could not give answer to the question of which of them may be the actual structure of real life PEDOT: HSulf crystallites. The hydrogen sulfate ions appeared to be small enough to fit in between PEDOT mers and their next nearest neighbors, but at the same time did not seem to be able to induce a motion of the PEDOT layers which would cause the pseudo-hexagonal structure to transit into the rectangular one.

\section{Crystalline structure of PEDOT: Sulf}

Once again, to determine whether sulfate ions can form a stable layer with the pseudo-hexagonal layout, the structure of PEDOT: OTf was used as a starting model for an MD simulation, with the triflate ions replaced with sulfate ions and the charge model changed to the appropriate one. Differently than in the case of PEDOT: HSulf, this system did not remain stable. The structure transited back into the earlier found system with a rectangular arrangement of sulfate ions over the course of the simulation, with a negligibly larger inter layer distance (11.1 A versus $10.9 \AA$ ). This was very likely to be caused by the small size of the sulfate ions, which allowed the PEDOT chains to move against each other without being hindered by the counter ions.

\section{CONCLUSIONS}

There are two major conclusions from carrying out this project, arising from the separation of the analysis into the structural and dynamical part.
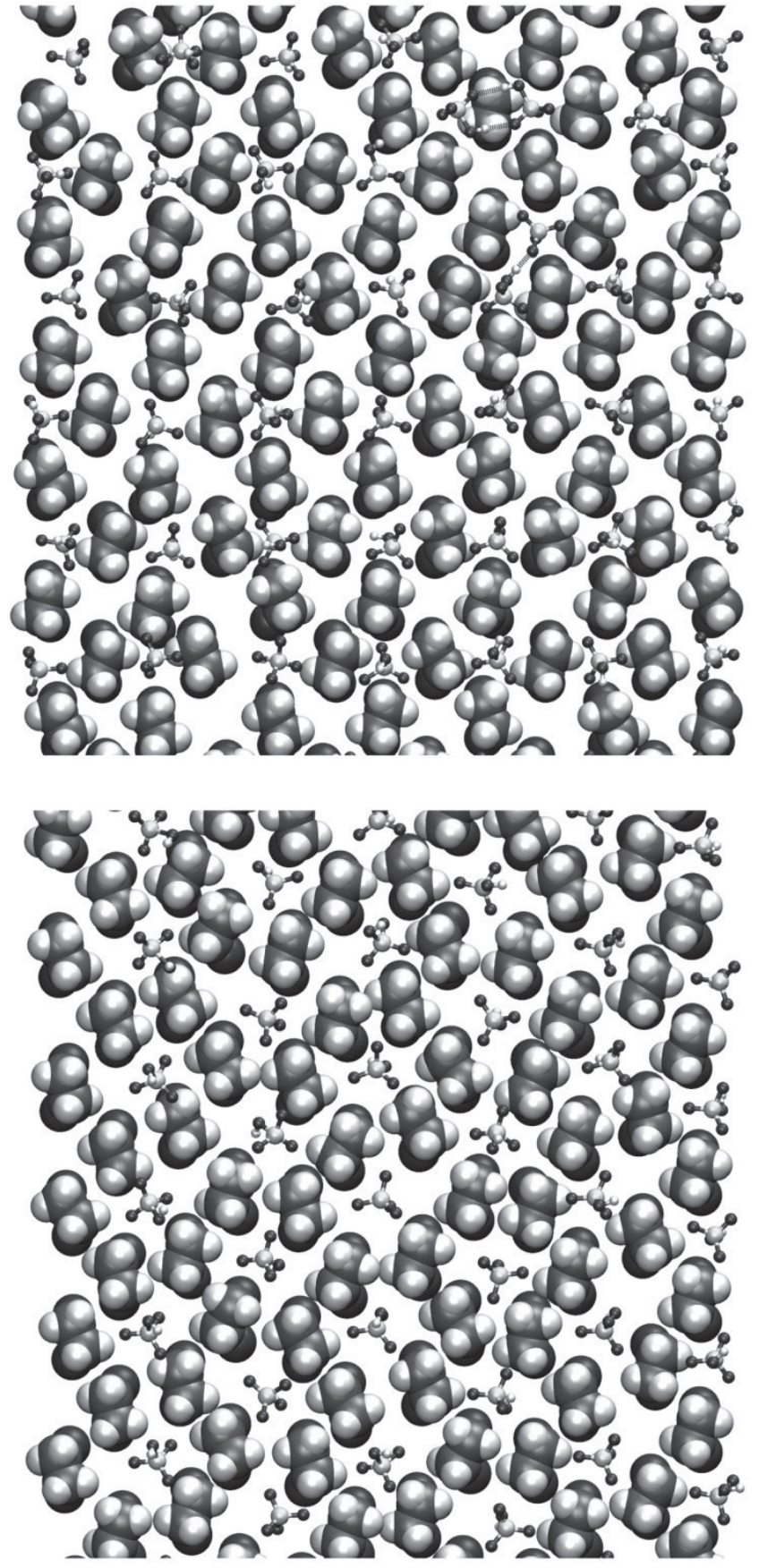

Fig. 11. The two stable structures of a hydrogen sulfate ion layer in the PEDOT:HSulf system; top: rectangular structure; bottom: pseudo-hexagonal structure; ball-and-stick models represent ions; spheres represent the $-\mathrm{O}-\mathrm{CH}_{2}-\mathrm{CH}_{2}-\mathrm{O}$ - fragments of PEDOT chains from the layer below

It must be concluded that the molecular dynamics simulations indicate that there is clearly a counter ion size effect on the preferred type of structure in doped PEDOT systems.

Very large anions, such as the tosylate ion, are large enough to form layers with a rectangular 2D layout which are capable of separating layers of stacked PEDOT chains from each other, preventing interaction between polymer layers. 
Large ions, yet not as much as the tosylate anion, such as the triflate anion, form layers which cannot isolate the stacked PEDOT layers from each other. The polymer layers are closer to each other and can interact with each other. The counter ions still form layers, but need to fit between the fragments of mers of the polymer. Because the polymer chains can rather easily slide against each other along their axes, a structure of distorted hexagonal "pockets" is established on either side of the polymer chain. This structure allows tight packing, since polymer layers can almost touch and the counter ions can settle inside these "pockets". The minimization of the electrostatic energy within ion layers leads to a pseudo-hexagonal structure.

Small counter ions such as the sulfate anion seem to not obstruct the polymer layers as much. They seem to be small enough to fit between mers and their next nearest neighbors, not enforcing the formation of the "pockets". Due to this, for small counter ions, their 2D structure is once again rectangular due to it being more optimal.

In the case of counter ions of intermediate size, such as the hydrogen sulfate anion, it could not be determined which of these two structures is the preferred one. Both appear stable in MD simulations, so the actual structure may be sensitive to external factors such as the structure in the amorphous or mesomorphic regions.

\section{REFERENCES}

[1] Massonnet N., Carella A., de Geyer A. et al.: Chemical Science 2015, 6, 412. https://doi.org/10.1039/C4SC02463J
[2] Gueye M.N., Carella A., Massonnet N. et al.: Chemistry of Materials 2016, 28, 3462. https://doi.org/10.1021/acs.chemmater.6b01035

[3] Gueye M.N., Carella A., Demadrille R., Simonato J.-P.: ACS Applied Materials and Interfaces 2017, 9, 27250. https://doi.org/10.1021/acsami.7b08578

[4] Xia Y., Sun K., Ouyang J.: Advanced Materials 2012, 24, 2436. https://doi.org/10.1002/adma.201104795

[5] Kozik T.: "Modeling of the structure of molecular and macromolecular systems using molecular dynamics and artificial intelligence as complementary methods", PhD thesis, AGH University of Science and Technology Cracow 2019.

[6] Piwowarczyk K.: "Molecular modeling in structural investigation of selected conducting polymers", $\mathrm{PhD}$ thesis, AGH University of Science and Technology Cracow 2011.

[7] Aasmundtveit K.E., Samuelsen E.J., Pettersson L.A.A.: Synthetic Metals 1999, 101, 561. https://doi.org/10.1016/S0379-6779(98)00315-4

[8] Kim E.-G., Bredas J.-L.: Journal of the American Chemical Society 2008, 130, 16880. https://doi.org/10.1021/ja806389b

[9] Śniechowski M., Kozik T., Niedźwiedź W., Łużny W.: Macromolecular Theory and Simulations 2016, 25, 328. https://doi.org/10.1002/mats.201600010

[10] Kozik T., Łużny W.: "Apllication of swarm intelligence algorithms for searching for the crystallographic structure of polymer systems", 58 Konwersatorium Krystalograficzne, Wrocław, 22-24 June 2016.

Received 16 III 2020. 